\title{
Effect of body-oriented psychological therapy on negative symptoms in schizophrenia: a randomized controlled trial
}

\author{
FRANK RÖHRICHT ${ }^{1 *}$ AND STEFAN PRIEBE ${ }^{2}$ \\ ${ }^{1}$ Consultant Psychiatrist, Honorary Senior Lecturer, Unit for Social \& Community Psychiatry, \\ Newham Centre for Mental Health; ${ }^{2}$ Professor of Social and Community Psychiatry, \\ Barts and the London School of Medicine, Queen Mary, University of London
}

\begin{abstract}
Background. In order to improve the treatment of medication-resistant negative symptoms in schizophrenia, new interventions are needed. Neuropsychological considerations and older reports in the literature point towards a potential benefit of body-oriented psychological therapy (BPT). This is the first randomized controlled trial specifically designed to test the effectiveness of manualized BPT on negative symptoms in chronic schizophrenia.

Method. Out-patients with DSM-IV continuous schizophrenia were randomly allocated to either BPT $(n=24)$ or supportive counseling $(\mathrm{SC}, n=21)$. Both therapies were administered in small groups in addition to treatment as usual (20 sessions over 10 weeks). Changes in negative symptom scores on the Positive and Negative Symptom Scale (PANSS) between baseline, post-treatment and 4-month follow-up were taken as primary outcome criteria in an intention-to-treat analysis.
\end{abstract}

Results. Patients receiving BPT attended more sessions and had significantly lower negative symptom scores after treatment (PANSS negative, blunted affect, motor retardation). The differences held true at 4-month follow-up. Other aspects of psychopathology and subjective quality of life did not change significantly in either group. Treatment satisfaction and ratings of the therapeutic relationship were similar in both groups.

Conclusions. BPT may be an effective treatment for negative symptoms in patients with chronic schizophrenia. The findings should merit further trials with larger sample sizes and detailed studies to explore the therapeutic mechanisms involved.

\section{INTRODUCTION}

Despite improvements in antipsychotic treatment schizophrenia patients often experience persistent symptoms and full remission is infrequent (Sheitman \& Lieberman, 1998). Andrews and co-workers concluded (2003) that current interventions avert only $13 \%$ of the burden of schizophrenia. Primary negative symptoms and the deficit syndrome appear to be particularly

\footnotetext{
* Address for correspondence: Dr Frank Röhricht, Academic Unit, Newham Centre for Mental Health, London E13 8SP, UK. (Email: Frank.Rohricht@elcmht.nhs.uk)
}

treatment-resistant (Arango et al. 2004), and there is a need to develop new, effective strategies to treat patients with negative symptoms of schizophrenia.

There are at least two reasons why bodyoriented psychotherapy (BPT), also referred to in the literature as 'body psychotherapy' (Staunton, 2002; Totton, 2003), may be worth studying in this context: positive reports in the literature on body-oriented interventions in schizophrenia, and neuropsychological considerations.

BPT refers back to a long tradition of bodyoriented interventions in psychiatry. At the 
beginning of the twentieth century the psychoanalysts Ferenczi and Reich were experimenting with non-verbal, body-oriented interventions to overcome perceived limitations of psychoanalytic practice. The earliest trials influenced by these ideas were undertaken by the Californian dance therapist Schoop. She started to work with hospitalized schizophrenia patients in 1959, and her 'body-ego technique' aimed to focus patients' attention 'on body posture and movement ... body-ego boundaries ... and reality contact and experience in movement' (May et al. 1963; Goertzel et al. 1965). A trial showed a significant improvement in patients treated with the technique, compared with controls, especially in affective contact, motility and general functioning (Goertzel et al. 1965).

Four further controlled studies - three of them randomized-compared other bodyoriented interventions with non-specific attention, music therapy or fitness training (Goertzel et al. 1965; Darby, 1970; Nitsun et al. 1974; Seruya, 1977). These studies were all conducted before 1980 and have serious methodological shortcomings, such as vaguely defined outcome criteria, no systematic assessment of psychopathology, no recording of medication, and no intention-to-treat analysis. Nevertheless, the results suggest favorable effects of the experimental treatments on a variety of outcome variables, including some indicators of negative symptoms.

The approach of body-oriented interventions is based on phenomenological findings (Priebe \& Röhricht, 2001; Röhricht \& Priebe, 2002) and the assumption that movement and emotional experiences are biologically and experientially associated. This is supported by close anatomical and functional links between the limbic system, particularly the extended amygdala, and the basal ganglia. It is also emphasized by Trimble's observation on how 'movement and emotion are linked in common speech (hence "a moving experience")' (1997: 114).

Two primary negative symptoms in particular lend themselves to body-oriented interventions: emotional withdrawal/affective blunting and motor retardation. Given their non-cognitive nature, they might be best targeted through non-verbal methods, combining sensory awareness techniques and emotional movement stimuli.
It is against this background that the first author of this paper defined a treatment manual for BPT with schizophrenia patients suffering from persistent negative symptoms. We report here the first randomized controlled trial of BPT for patients with schizophrenia in recent history. The trial tested the hypothesis that BPT is effective in reducing negative symptoms in out-patients with schizophrenia. To control for the influence of non-specific attention and structured group activities BPT was compared with supportive counseling (SC).

\section{METHOD}

\section{Recruitment and procedure}

The study was conducted in East London, UK. Patients were recruited by referrals from community mental health services. The study was approved by the North East London Strategic Health Authority Ethics Committee and written informed consent was obtained from all patients before trial entry.

We applied the following selection criteria: age 20-55 years; an established diagnosis of schizophrenia according to DSM-IV, with at least two episodes with acute psychotic symptoms; time since last in-patient treatment more than 1 month (currently out-patient); suffering from persistent symptoms of schizophrenia for at least 6 months with a high degree of negative symptoms at baseline, i.e. Positive and Negative Symptoms Scale (PANSS) subscore 'Negative' $\geqslant 20$ and/or one of the 'Anergia' items ('emotional withdrawal', 'motor retardation' or 'blunted affect') $\geqslant 6$ $(6=$ severe $)$; stable medication prior to entering the study. Exclusion criteria were: evidence of organic brain disease; severe or chronic physical illness; and substance misuse as primary diagnosis. An experienced psychiatrist, blind to the allocated treatment, carried out all screening, baseline and outcome assessments; the rater was trained in the use of assessment instruments. All patients referred to the project were offered an appointment for a screening interview to establish whether selection criteria were met. Suitable patients were then further assessed (details below) within the same interview. Eligible patients were randomly allocated to one of the two treatment conditions (BPT or $\mathrm{SC}$, both in addition to treatment as usual) 
following the opening of a sealed envelope by the project co-ordinator, who had no involvement in data collection or assessments. This was carried out in blocks: once a sufficient number of patients had been recruited to the study to fill one treatment group in each condition, the recruited patients were randomly allocated.

\section{Treatment conditions}

All patients in both treatment arms received psychological group treatments in addition to the usual care provided by community psychiatric services (TAU). Treatment plans were not substantially altered during the trial period. In both conditions, BPT and SC, the group size was limited to a maximum of eight patients, and the aim was to provide 20 sessions of $60-90$ minutes each over a period of 10 weeks.

The therapists providing treatment in the study were otherwise not involved in the patients' care. A part-time dance movement therapist conducted BPT. Two nurse therapists, also with previous training and experience in providing psychological therapies for schizophrenia patients, delivered SC. All therapists had many years' experience of working with patients suffering from schizophrenia and attended specific training sessions before the trial. Later they received three supervision sessions each to ensure adherence to the given treatment manual (on the basis of written records of each session).

\section{Body-oriented psychological therapy (BPT)}

Different schools of body-oriented psychotherapeutic interventions have developed, but various authors acknowledge the underlying coherence and substantial overlap in the applied intervention strategies (e.g. Guimon, 1997; Staunton, 2002; Totton, 2003). The treatment manual used in this study was defined (by the first author) based on the available evidence and aimed to integrate different techniques (e.g. Krietsch \& Heuer, 1997; Scharfetter, 1999) into a clinically focused and syndrome-specific method (for full description see Röhricht, 2000).

The protocol of the manual was designed to achieve the following aims:

(1) to overcome communication barriers through the introduction of non-verbal techniques;
(2) to refocus cognitive and emotional awareness towards the body (physical reality, co-ordination and orientation in space);

(3) to stimulate activity and emotional responsiveness;

(4) to promote exploration of self-potentials, focusing on body strength and capability, experiencing the body as a source of creativity, reliability, pleasure and selfexpression;

(5) to modify dysfunctional self-perception;

(6) to address common psychopathological features such as boundary loss, somatic depersonalization, and body schema disturbances.

BPT was delivered within a format of defined sections as follows (intervention examples given for each section):

(A) Opening circle: checking in: 'How do you feel, how does your body feel (i.e. warm, cold, tense, floppy)? Describe your level of energy; where is the centre of your body-awareness?' Sitting in a circle on the floor and engaging in simple warm-up activities and communication tasks with props such as soft balls, balloons and beanbags.

(B) Warm up section: standing in a circle, continuation of warm-up using different body parts and different qualities of movement, e.g. swings, stretches, jumps. Grounding, bodycentering and body awareness techniques/ exercises and movements, focusing on basic physiological functions such as breathing and pulsation. Travelling movements including different kinds of walks in different directions, at different speeds and with different qualities, e.g. brisk, purposeful walk in contrast to lethargic walk as well as crawling, jumping, turning; exploring the dimensions of space within and outside the body.

(C) Structured task section: exploring immediate vicinity from small to big and in all three dimensions; demarcating own boundaries with props, e.g. rope. Identifying a partner, defining demarcation of own boundaries in response to feedback. Mirroring exercises copying each others' movements; leading and following from a stationary position and then travelling with the purpose of exploring the body-ego as consistent, self-evident and active; exploring emotionally equivalent movements, 
i.e. stamping, stroking, hiding away, defending. Creating body image sculptures on paper or in partners and comparing internal with external body-schema.

(D) Creative movement section: back in group circle. Group mirroring with each participant having an opportunity to initiate movement phrases in the group based either on pure movement invention stimulated by rhythmic music, or with a concrete theme like different sports themes, or related to feelings/ opposites. Creating group sculptures. Reflecting on how this feels: 'Can you engage in these movement exercises?', 'Do you feel stress/ anxiety/discomfort/pleasure/confidence when leading and/or following?'

(E) Closing circle: reflecting on group experiences, energy levels, re-focusing on self with simple body-oriented exercises such as selftouch, verbal integration.

\section{Supportive counseling (SC)}

Basic principles of the method are described elsewhere (Tarrier et al. 1993; Valmaggia et al. 2005). In this study, the therapist focused on individual difficulties and corresponding problem-solving strategies regarding the core negative symptoms. The therapist initially facilitated a safe and supportive atmosphere amongst group participants; in the next step patients were given the opportunity to talk about specific difficulties in relation to lack of motivation, difficulties initiating activities, and lack of emotional responsiveness; the group then engaged in discussing their experiences, trying to identify the impact of the symptoms on their lives and possible contributing factors to the problems; this was followed by the therapists' emphasis on examples of good practice, i.e. well established coping strategies, as well as creative attempts to identify possible solutions related to individual difficulties, followed by verbal closure, integrating the different aspects of the session.

\section{Sample size}

In this exploratory trial, the power calculation was based on the aim to detect a moderate to large effect size, comparable to effects in published trials on other forms of psychotherapy for persistent symptoms of schizophrenia (Kuipers et al. 1997; Durham et al. 2003). A trial with a total sample of 40-60 patients would provide $55 \%$ power of detecting an effect size of $0 \cdot 6$, and $81 \%$ power for an effect size of 0.8 with a two-tailed significance level of 0.05 (Cohen, 1992; Tarrier et al. 1993).

\section{Primary and secondary outcome assessments}

Patients were assessed prior to and at the end of treatment as well as after a 4-month followup period. The work of the therapists and the assessing researcher were kept strictly separate in order to ensure blindness of the assessor, and patients were requested not to reveal any details of their treatment during post-treatment assessments up to the end of the follow-up interview, when qualitative data was collected.

The primary outcome measure of the study was the level of negative symptoms as rated on the corresponding subscale of the PANSS (Kay et al. 1987). We specifically assessed changes in 'affective blunting' and 'decreased spontaneous movement' (psychomotor retardation), because these symptoms are regarded as 'core negative symptoms' (Liddle, 2000) of chronic schizophrenia. Since negative symptoms may be secondary to extrapyramidal side-effects of antipsychotic medication, these were recorded using the Simpson-Angus Extrapyramidal Symptom Scale (EPS; Simpson \& Angus, 1970). Antipsychotic medication was documented as chlorpromazine-equivalent (Atkins et al. 1997; BMA, 2003) at all three points in order to assess the impact on treatment outcome.

Other psychopathological symptoms (PANSS positive and PANSS general), and subjective quality of life (SQOL) were assessed at three time-points as secondary outcome measures.

The Manchester Short Assessment of Quality of Life (MANSA; Priebe et al. 1999) was used to assess SQOL (providing a mean score of satisfaction ratings in 12 life domains, each ranging on a Likert scale of $1=$ 'could not be worse' to $7=$ 'could not be better').

Patients' satisfaction with treatment was measured post-treatment and at follow-up on the Client's Assessment of Treatment Scale (CAT; Priebe et al. 1995), comprising seven 11 -point rating scales ranging from $0=$ extreme negative answer to $10=$ extreme positive answer on different aspects of treatment. At follow-up the same scale was administered to assess retrospective satisfaction with treatment. 
We also assessed the quality of the therapeutic relationship after treatment and at follow-up as a non-specific and potentially mediating factor. Patients rated the Helping Alliance Scale (HAS; Priebe \& Gruyters, 1993), which consists of five Likert-type items. The ratings are summarized, with higher scores indicating a better quality of the relationship.

\section{Analysis}

All data were analyzed using the Statistical Package for the Social Sciences version $10 \cdot 1$. for Windows (SPSS Inc., Chicago, IL, USA). Analyses were conducted on an intentionto-treat basis. Differences in negative symptom scores between the experimental intervention and control groups were tested using analysis of covariance (ANCOVA), with baseline scores as covariates. ANCOVA was also conducted on the mean of satisfaction ratings in the MANSA post-treatment and follow-up, with corresponding baseline scores as a covariate. Another analysis examined the proportion of patients in each treatment group who showed an improvement between baseline and posttreatment of $25 \%$ or greater in negative symptom scores.

Changes of medication during participation in the trial were recorded as both changes in mean daily equivalents of chlorpromazine and changes from typical to atypical antipsychotics. In order to examine the impact of dosage of antipsychotic medication and extrapyramidal symptoms on negative symptoms, ANCOVA analysis of variance was repeated with the chlorpromazine-equivalents of antipsychotic medication and the EPS scale total score as covariates. Furthermore it was intended to analyze the data based on group allocation as follows: no change of medication, change from typical to typical antipsychotic, change from typical to atypical antipsychotic. Patients' ratings of treatment and the therapeutic relationship were analyzed using $t$ tests.

\section{RESULTS}

\section{Description of sample}

A total of 67 patients were referred for inclusion in the study, 55 of whom fulfilled the inclusion criteria. Of these, 45 consented and were randomized to the treatment conditions. In total, four groups of patients were treated in each condition. The detailed flow diagram is shown in Fig. 1.

Demographic and clinical characteristics of the study sample $(n=45)$ are shown in Table 1.

None of the variables showed significant statistical difference between the two groups. The sample consisted mainly of middle-aged, single, unemployed individuals, and participants had a long history of mental illness.

The two groups differed significantly with respect to the average number of therapy sessions attended: BPT $(n=11 \cdot 3$, s.D. $=6 \cdot 0)$; SC $(n=4 \cdot 5$, S.D. $=4 \cdot 8) ; t=4 \cdot 0, \mathrm{df}=43, p<0 \cdot 001$. Dosages of antipsychotic medication as well as extrapyramidal symptom scale scores did not differ significantly between the two groups (Table 2).

\section{Outcome measures}

Mean scores of the psychopathology outcome measures and SQOL measures from baseline to follow-up are shown in Table 3. The two groups showed no significant differences in psychopathological scores at baseline.

\section{Changes of negative symptom severity from baseline}

Controlling for baseline scores, the ANCOVA of patients' negative symptom scores showed a significant effect of the experimental intervention: patients treated with BPT had significantly lower symptom scores after treatment (PANSS negative: $F=5 \cdot 0, p=0.031$; blunted affect: $F=10.8, p=0.002$; motor retardation: $F=4.7, p=0.035$ ) and at follow-up (PANSS negative: $F=7 \cdot 0, \quad p \leqslant 0.015$; blunted affect: $F=5.6, p=0.026$; motor retardation: $F=7 \cdot 7$, $p=0.011)$.

The number of patients with symptom reduction of $20 \%$ or more (range 20-46\%) from baseline score was significantly higher in the BPT group (PANSS negative: $n=12 / 50 \%$ versus $n=4 / 21 \%$ ). When repeating ANCOVA with chlorpromazine-equivalents of antipsychotic medication and the EPS scale total score as additional covariates, the results of the analyses were not substantially affected by these covariates. Thus, differences in treatment effects on negative symptoms were not influenced by extrapyramidal side-effects or level of antipsychotic medication as measured in this trial. 


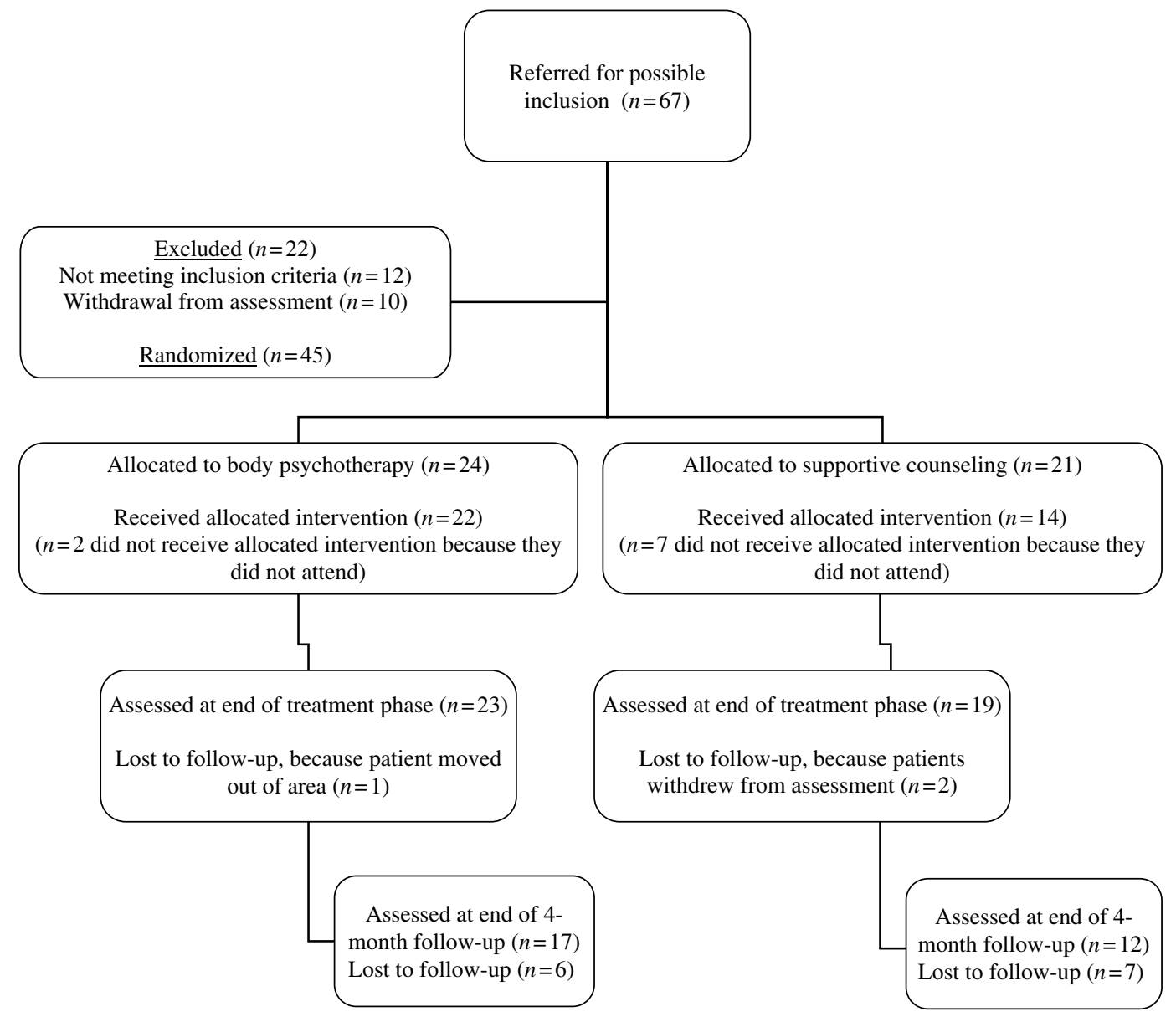

FIG. 1. Study flow diagram.

Changes of antipsychotic substances from baseline to post-treatment occurred only in seven patients (BPT, $n=2 ; \mathrm{SC}, n=5)$, not allowing for analysis of variance as intended. A case-by-case analysis showed that in four patients medication was changed from one to another atypical antipsychotic, two patients were changed from typical to atypical antipsychotic, and one patient from atypical to typical antipsychotic. These changes were not associated with more or less favorable treatment outcome.

\section{Other outcome measures}

Other psychopathology symptom scores (PANSS positive, general, and total) as well as SQOL scores did not differ significantly, either within or between groups.
Patients' assessment of treatment was broadly positive: the mean CAT score did not differ between groups after treatment $(\mathrm{BPT}:$ mean $=6 \cdot 8$, S.D. $=2.0 ; \mathrm{SC}:$ mean $=6.4$, S.D. $=1.9)$ and at follow-up (BPT: mean $=7 \cdot 3$, s.D. $=1.9 ; \quad \mathrm{SC}$ : mean $=6 \cdot 7$, s.D. $=1 \cdot 8$ ). Equally, patients' ratings of the therapeutic relationship was generally appreciative and did not differ between groups after treatment (BPT: mean $=7 \cdot 2$, S.D. $=1 \cdot 9$; SC: mean $=6 \cdot 6$, S.D. $=1 \cdot 8)$ and at follow-up (BPT: mean $=7 \cdot 1$, S.D. $=2 \cdot 1 ; \mathrm{SC}:$ mean $=7 \cdot 1$, S.D. $=1 \cdot 6)$.

\section{DISCUSSION}

\section{Overall findings}

BPT was administered without worsening of positive, florid psychotic symptoms. It was more 
Table 1. Demographic data on participants who entered the trial

\begin{tabular}{lcc}
\hline \hline & BPT $(n=24)$ & SC $(n=21)$ \\
\hline Gender, F/M & $12 / 12$ & $11 / 10$ \\
Age, yr (mean/s.D.) & $38 \cdot 8 / 9 \cdot 3$ & $37 \cdot 7 / 9 \cdot 5$ \\
Duration of illness, yr (mean/s.D.) & $12 \cdot 1 / 10 \cdot 5$ & $10 \cdot 8 / 7 \cdot 3$ \\
No. of previous hospitalizations & $3 \cdot 7 / 2 \cdot 8$ & $4 \cdot 4 / 3 \cdot 8$ \\
(mean/s.D.) & & \\
Age at leaving full-time education, yr & $16 \cdot 4 / 2 \cdot 3$ & $17 \cdot 0 / 2 \cdot 2$ \\
(mean/s.D.) & & \\
Accommodation & & \\
Lives alone, $n(\%)$ & $12(50 \cdot 0)$ & $9(42 \cdot 9)$ \\
With parents, $n(\%)$ & $5(20 \cdot 8)$ & $8(38 \cdot 1)$ \\
With partner, $n(\%)$ & $4(16 \cdot 7)$ & $4(19 \cdot 0)$ \\
With child $>18, n(\%)$ & $3(12 \cdot 5)$ & $0(0)$ \\
Employment status & & \\
$\quad$ Unemployed, $n(\%)$ & $21(87 \cdot 5)$ & $19(90 \cdot 5)$ \\
Sheltered work, $n(\%)$ & $2(8 \cdot 4)$ & $1(4 \cdot 8)$ \\
Employed, $n(\%)$ & $1(4 \cdot 1)$ & $1(4 \cdot 8)$ \\
\hline \hline
\end{tabular}

BPT, Body-oriented psychological therapy; SC, supportive counseling.

effective in improving persistent and medication-resistant primary negative symptoms than SC, when given in addition to treatment as usual.

The findings did not suggest an influence of potentially confounding factors, i.e. antipsychotic medication, extrapyramidal symptoms, improvement of positive symptoms, on the different treatment effect in the two groups. Both groups showed similar treatment satisfaction and ratings of therapeutic relationships. The effect of BPT, therefore, cannot be explained by non-specific effects as reflected in treatment satisfaction and the quality of the therapeutic relationship. Applying the criterion of $20 \%$ reduction on symptom scale scores as a measure of clinically significant change (as suggested by Rector et al. 2003), a significantly higher number of patients in the BPT group $(50 \%)$ achieved this degree of response to the treatment.

\section{Limitations of the study}

This was an exploratory trial with a small sample size. A single therapist administered BPT, and it remains unclear whether the effect can be replicated across different therapists and in other samples and settings. However, the manualization should help to reduce variation in the delivery of BPT.
Table 2. Antipsychotic medication (chlorpromazine-equivalent) and extrapyramidal symptom scale scores

\begin{tabular}{|c|c|c|c|c|c|c|}
\hline & \multicolumn{3}{|c|}{ BPT } & \multicolumn{3}{|c|}{$\mathrm{SC}$} \\
\hline & $n$ & Mean & S.D. & $n$ & Mean & S.D. \\
\hline \multicolumn{7}{|c|}{ Chlorpromazine-equivalents of antipsychotic medication } \\
\hline At baseline & 24 & $497 \cdot 9$ & $289 \cdot 1$ & 21 & $440 \cdot 5$ & $324 \cdot 8$ \\
\hline Post-treatment & 24 & $506 \cdot 5$ & $296 \cdot 8$ & 19 & $411 \cdot 4$ & $241 \cdot 1$ \\
\hline At follow-up & 17 & $453 \cdot 6$ & $324 \cdot 8$ & 12 & $529 \cdot 5$ & $295 \cdot 2$ \\
\hline \multicolumn{7}{|c|}{ Extrapyramidal symptom scale total score } \\
\hline At baseline & 24 & $1 \cdot 5$ & $1 \cdot 7$ & 21 & $1 \cdot 4$ & 1.9 \\
\hline Post-treatment & 24 & $1 \cdot 3$ & $1 \cdot 6$ & 19 & 1.6 & $2 \cdot 2$ \\
\hline At follow-up & 17 & $1 \cdot 4$ & $1 \cdot 8$ & 12 & $1 \cdot 5$ & $2 \cdot 0$ \\
\hline
\end{tabular}

BPT, Body-oriented psychological therapy; SC, supportive counseling.

There was a high drop-out rate in the control group. Some of the clinical improvement may therefore be attributed to better treatment adherence in the experimental group and nonspecific effects of more attention and activities. However, we did not find a difference in indicators of non-specific effects between the two groups in an intention-to-treat analysis. Also, the better adherence of patients to BPT shows a relatively good acceptance of the experimental treatment, which may be regarded as a positive effect of BPT itself and facilitate its use in practice.

\section{Comparison with other trials on negative symptoms}

Compared with results from trials on the efficacy of atypical antipsychotics on negative symptoms (reviews Leucht et al. 1999; Möller, 2000; Chakos et al. 2001), the results of this study appear encouraging. A review of Chakos et al. (2001) found effects of clozapine, olanzapine or risperidone on negative symptoms with an improvement of between $3 \%$ and $15 \%$, i.e. lower than the mean reduction of $20-25 \%$ in this study. Volavka et al. (2002) directly compared clozapine, olanzapine, risperidone and haloperidol in the treatment of chronic schizophenia. Only in patients treated with clozapine was a significant improvement in negative symptoms identified after 8 weeks - comparable to the findings in this study.

Only a few studies have so far tested cognitive-behavioural therapy (CBT) targeting 
Table 3. Clinical outcome measures (ANCOVAs, adjusted for baseline score)

\begin{tabular}{|c|c|c|c|c|c|c|c|}
\hline & \multicolumn{3}{|c|}{ BPT group } & \multicolumn{3}{|c|}{ SC group } & \multirow[b]{2}{*}{ Difference $(95 \% \mathrm{CI})$} \\
\hline & $n$ & Mean & S.D. & $n$ & Mean & S.D. & \\
\hline \multicolumn{8}{|l|}{ PANSS negative } \\
\hline At baseline & 24 & $23 \cdot 4$ & $4 \cdot 1$ & 21 & $24 \cdot 6$ & $5 \cdot 1$ & \\
\hline Post-treatment & 24 & $18 \cdot 9$ & $4 \cdot 3$ & 19 & $23 \cdot 3$ & $7 \cdot 4$ & $3.46(0.34 \text { to } 6.58)^{*}$ \\
\hline At follow-up & 15 & $18 \cdot 2$ & $2 \cdot 5$ & 12 & $23 \cdot 2$ & $6 \cdot 3$ & $4.49(1.09 \text { to } 7.89)^{*}$ \\
\hline \multicolumn{8}{|l|}{ Blunted affect } \\
\hline At baseline & 24 & $5 \cdot 0$ & $0 \cdot 9$ & 21 & $4 \cdot 9$ & $0 \cdot 8$ & \\
\hline Post-treatment & 24 & $3 \cdot 7$ & $1 \cdot 0$ & 19 & $4 \cdot 7$ & 1.5 & $1.2(0.39 \text { to } 1.64)^{*}$ \\
\hline At follow-up & 15 & $3 \cdot 5$ & $1 \cdot 1$ & 12 & $4 \cdot 6$ & $1 \cdot 2$ & $1.1(0.14 \text { to } 1.97)^{*}$ \\
\hline \multicolumn{8}{|l|}{ Motor retardation } \\
\hline At baseline & 24 & $3 \cdot 2$ & $1 \cdot 2$ & 21 & $3 \cdot 3$ & $1 \cdot 0$ & \\
\hline Post-treatment & 24 & $2 \cdot 4$ & $1 \cdot 0$ & 19 & $3 \cdot 2$ & $1 \cdot 2$ & $1.0(0.05 \text { to } 1.40)^{*}$ \\
\hline At follow-up & 15 & $2 \cdot 3$ & $1 \cdot 0$ & 12 & $3 \cdot 5$ & $1 \cdot 2$ & $0.7(0.25 \text { to } 1.75)^{*}$ \\
\hline \multicolumn{8}{|l|}{ PANSS positive } \\
\hline At baseline & 24 & $16 \cdot 5$ & $5 \cdot 4$ & 21 & $13 \cdot 1$ & $6 \cdot 8$ & \\
\hline Post-treatment & 24 & $15 \cdot 3$ & $5 \cdot 1$ & 19 & $12 \cdot 8$ & $5 \cdot 4$ & $0.20(-1.90$ to 2.32$)$ \\
\hline At follow-up & 15 & $14 \cdot 8$ & $5 \cdot 5$ & 12 & $12 \cdot 3$ & $4 \cdot 0$ & $0.29(-0.2 .33$ to $2 \cdot 90)$ \\
\hline \multicolumn{8}{|l|}{ PANSS general } \\
\hline At baseline & 24 & $39 \cdot 1$ & $8 \cdot 4$ & 21 & $38 \cdot 6$ & $10 \cdot 8$ & \\
\hline Post-treatment & 24 & $37 \cdot 2$ & $8 \cdot 7$ & 19 & $37 \cdot 2$ & $9 \cdot 9$ & $0.24(-3.89$ to 4.38$)$ \\
\hline At follow-up & 15 & $37 \cdot 3$ & $5 \cdot 4$ & 12 & $38 \cdot 9$ & $9 \cdot 6$ & $2.04(-3.60$ to 7.69$)$ \\
\hline \multicolumn{8}{|l|}{ PANSS total sum } \\
\hline At baseline & 24 & $79 \cdot 0$ & $13 \cdot 9$ & 21 & $76 \cdot 3$ & $21 \cdot 1$ & \\
\hline Post-treatment & 24 & $71 \cdot 4$ & $15 \cdot 7$ & 19 & 71.9 & $20 \cdot 9$ & $5 \cdot 78(-4 \cdot 26$ to $15 \cdot 82)$ \\
\hline At follow-up & 15 & $70 \cdot 3$ & $10 \cdot 0$ & 12 & $74 \cdot 4$ & $17 \cdot 1$ & $3.35(-4.95$ to 11.65$)$ \\
\hline \multicolumn{8}{|l|}{ MANSA total sum } \\
\hline At baseline & 21 & $4 \cdot 1$ & $0 \cdot 9$ & 18 & $4 \cdot 1$ & $0 \cdot 7$ & \\
\hline Post-treatment & 21 & $4 \cdot 1$ & $0 \cdot 7$ & 18 & $4 \cdot 1$ & $0 \cdot 8$ & $0 \cdot 10(-0.26$ to 0.46$)$ \\
\hline At follow-up & 15 & $4 \cdot 3$ & $0 \cdot 5$ & 12 & 3.9 & $0 \cdot 8$ & $-0.27(0.11$ to 0.72$)$ \\
\hline
\end{tabular}

PANSS, Positive and Negative Symptom Scale; MANSA, Manchester Short Assessment of Quality of Life; BPT, Body-oriented psychological therapy; SC, supportive counseling.

$* p<0 \cdot 05$.

negative symptoms in schizophrenia (Tarrier, 2005). Rector \& Beck (2001) identified three studies with medium to large treatment effects of CBT on negative symptoms as compared with routine care or supportive therapy. Sensky et al. (2000) reported a significant improvement of negative symptoms in both CBT and the non-specific befriending control condition, which was sustained after 9 months only in the CBT group. Rector et al. (2003) remarked that these changes might have been secondary to changes in positive and/or depressive symptoms, a concern that does not apply to the findings of this study. In their own study, Rector et al. (2003) found that $61 \%$ of patients with persistent symptoms receiving CBT were regarded as treatment 'responders' compared with $31 \%$ in 'enriched treatment as usual', and the effects were not attributable to changes in positive symptoms and/or depression. However, the baseline scores for negative symptoms were significantly lower than in our study. In various trials (Tarrier et al. 1993; Sensky et al. 2000; Tarrier et al. 2000) CBT has been associated with lower drop-out rates than the control conditions, as has BPT in our study.

There is currently no evidence suggesting that other non-pharmacological therapies (family interventions, social skills training, cognitive remediation, psychoeducation, assertive community treatment) have consistent effects on negative symptoms in schizophrenia (Bustillo et al. 2001; Pilling et al. 2002; Turkington et al. 2004).

\section{CONCLUSIONS}

In this exploratory trial of BPT we targeted a highly selective patient group with marked and dominating negative symptoms of chronic 
schizophrenia. BPT was accepted by patients and associated with a favorable effect. The effect size was substantial and at least as high as those reported in the literature for antipsychotic medication and CBT. The findings may merit further trials with larger sample sizes and detailed studies to explore the therapeutic mechanisms involved. Such studies might lead to amendments to the approach and manual of BPT to optimise the therapeutic effect.

If the effects can be replicated, it may be tested whether BPT can be combined with other psychological treatments such as CBT to achieve an increased overall effect or whether the different strategies should rather be seen as alternatives, possibly for different subgroups of patients.

\section{ACKNOWLEDGEMENTS}

The study was supported by the East London $\&$ The City Mental Health Trust and an unconditional grant by Pfizer and Wyeth. We are grateful to Dr Iris Suzuki for her help with data collection and Dr Michael Dewey for his statistical advice.

\section{DECLARATION OF INTEREST}

None.

\section{REFERENCES}

Andrews, G., Sanderson, K., Corry, J., Issakidis, C. \& Lapsley, H. (2003). Cost-effectiveness of current and optimal treatment for schizophrenia. British Journal of Psychiatry 183, 427-435.

Arango, C., Buchanan, R. W., Kirkpatrick, B. \& Carpenter, W. T. (2004). The deficit syndrome in schizophrenia: implications for the treatment of negative symptoms. European Psychiatry 19, 21-26.

Atkins, M., Burgess, A., Bottomley, C. \& Riccio, M. (1997). Chlorpromazine equivalents: a consensus of opinion for both clinical and research applications. Psychiatric Bulletin 21, 224-226.

BMA \& RPSGB (2003). British National Formulary BNF 46. British Medical Association \& Royal Pharmaceutical Society of Great Britain: London.

Bustillo, J., Lauriello, J., Horan, W. \& Keith, S. (2001). The psychosocial treatment of schizophrenia: an update. American Journal of Psychiatry 158, 163-175.

Chakos, M., Lieberman, J., Hoffman, E., Bradford, D. \& Sheitman, B. (2001). Effectiveness of second-generation antipsychotics in patients with treatment-resistant schizophrenia: a review and meta-analysis of randomized trials. American Journal of Psychiatry 158, 518-526.

Cohen, J. (1992). A power primer. Psychological Bulletin 112, 155-159.

Darby, J. A. (1970). Alteration of some body image indexes in schizophrenia. Journal of Consulting and Clinical Psychology 35 , 116-121.
Durham, R. C., Guthrie, M., Morton, R. V., Reid, D. A., Treliving, L. R., Fowler, D. \& MacDonald, R. R. (2003). Tayside-Fife clinical trial of cognitive-behavioural therapy for medication-resistant psychotic symptoms. Results to 3-month follow-up. British Journal of Psychiatry 182, 303-311.

Goertzel, V., May, P. R. A., Salkin, J. \& Schoop, T. (1965). Body-ego technique: an approach to the schizophrenic patient. Journal of Nervous and Mental Disease 141, 53-60.

Guimon, J. (1997). The Body in Psychotherapy. Karger: Basel.

Kay, S. R., Fiszbein, A. \& Opler, L. A. (1987). The positive and negative syndrome scale (PANSS) for schizophrenia. Schizophrenia Bulletin 13, 261-276.

Krietsch, S. \& Heuer, B. (1997). Movement Therapy with Schizophrenia Patients [in German]. Gustav Fischer: Lübeck, Stuttgart, Jena, Ulm.

Kuipers, E., Garety, P., Fowler, D., Dunn, G., Bebbington, P., Freeman, D. \& Hadley, C. (1997). London-East Anglia randomised controlled trial of cognitive-behavioural therapy for psychosis. I: Effects of the treatment phase. British Journal of Psychiatry 171, 319-327.

Leucht, S., Pitschel-Walz, G., Abraham, D. \& Kissling, W. (1999). Efficacy and extrapyramidal side-effects of the new antipsychotics olanzapine, quetiapine, risperidone, and sertindole compared to conventional antipsychotics and placebo. A meta-analysis of randomized controlled trials. Schizophrenia Research 35, 51-68.

Liddle, P. F. (2000). Descriptive clinical features of schizophrenia. In New Oxford Textbook of Psychiatry, vol. 1 (ed. M. G. Gelder, J. J. Lopez-Ibor and N. C. Andreasen), pp. 571-576. Oxford University Press: New York.

May, P. R. A., Wexler, M., Salkin, J. \& Schoop, T. (1963). Nonverbal techniques in the re-establishment of body image and self identity - a preliminary report. Research Report 16, 68-82.

Möller, H. J. (2000). Definition, psychopharmacological basis and clinical evaluation of novel/atypical neuroleptics: methodological issues and clinical consequences. World Journal of Biological Psychiatry 1, 75-91.

Nitsun, M., Stapleton, J. H. \& Bender, M. P. (1974). Movement and drama therapy with long-stay schizophrenics. British Journal of Medical Psychology 47, 101-119.

Pilling, S., Bebbington, P., Kuipers, E., Garety, P., Geddes, J. Martindale, B., Orbach, G. \& Morgan, C. (2002). Psychological treatments in schizophrenia: II. Meta-analysis of randomized controlled trials of social skills training and cognitive remediation. Psychological Medicine 32, 783-791.

Priebe, S. \& Gruyters, T. (1993). The role of the helping alliance scale in psychiatric community care. Journal of Nervous and Mental Disease 181, 552-557.

Priebe, S., Gruyters, T., Heinze, M., Hoffmann, C. \& Jäkel, A. (1995). Subjective criteria in psychiatric care - methods of assessment for research and routine care [in German]. Psychiatrische Praxis 22, $140-144$.

Priebe, S., Huxley, P., Knight, S. \& Evans, S. (1999). Application and results of the Manchester Short Assessment of Quality of Life (MANSA). International Journal of Social Psychiatry 45, 7-12.

Priebe, S. \& Röhricht, F. (2001). Specific body image pathology in schizophrenia. Psychiatry Research 101, 289-301.

Rector, N. A. \& Beck, A. T. (2001). Cognitive behavioral therapy for schizophrenia: an empirical review. Journal of Nervous and Mental Disease 189, 278-287.

Rector, N. A., Seeman, M. V. \& Segal, Z. V. (2003). Cognitive therapy for schizophrenia: a preliminary randomized controlled trial. Schizophrenia Research 63, 1-11.

Röhricht, F. (2000). Body-oriented Psychotherapy in Mental Illness. A Manual for Research and Practice [in German]. Hogrefe: Göttingen, Bern, Toronto, Seattle.

Röhricht, F. \& Priebe, S. (2002). Do cenesthesias and body image aberration characterize a subgroup in schizophrenia? Acta Psychiatrica Scandinavica 105, 276-282.

Scharfetter, C. (1999). Schizophrenic ego disorders - argument for body-including therapy [in German]. Schweizer Archiv für Neurologie und Psychiatrie 150, 11-15. 
Sensky, T., Turkington, D., Kingdon, D., Scott, J. L., Scott, J., Siddle, R., O'Caroll, M. \& Barnes, T. R. (2000). A randomized controlled trial of cognitive-behavioral therapy for persistent symptoms in schizophrenia resistant to medication. Archives of General Psychiatry 57, 165-172.

Seruya, B. B. (1977). The effects of training on body-size estimation of schizophrenics [Dissertation]. Abstracts International 38, 1421.

Sheitman, B. B. \& Lieberman, J. A. (1998). The natural history and pathophysiology of treatment resistant schizophrenia. Journal of Psychiatric Research 32, 143-150.

Simpson, G. M. \& Angus, J. W. S. (1970). A rating scale for extrapyramidal side effects. Acta Psychiatrica Scandinavica 45, 11-19.

Staunton, T. (2002). Body Psychotherapy. Brunner-Routledge: East Sussex.

Tarrier, N. (2005). Cognitive behaviour therapy for schizophrenia - a review of development, evidence and implementation. Psychotherapy Psychosomatics 74, 136-144.

Tarrier, N., Beckett, R., Harwood, S., Baker, A., Yusupoff, L. \& Ugarteburu, I. (1993). A trial of two cognitive-behavioural methods of treating drug-resistant residual psychotic symptoms in schizophrenic patients: I. Outcome. British Journal of Psychiatry 162, 524-532.
Tarrier, N., Kinney, C., McCarthy, E., Humphreys, L., Wittkowski, A. \& Morris, J. (2000). Two-year follow-up of cognitivebehavioral therapy and supportive counseling in the treatment of persistent symptoms in chronic schizophrenia. Journal of Consulting and Clinical Psychology 68, 917-922.

Totton, N. (2003). Body Psychotherapy: An Introduction. Open University Press: Maidenhead-Philadelphia.

Trimble, M. R. (1997). Biological Psychiatry (2nd edn). Wiley: Chichester.

Turkington, D., Dudley, R., Warman, D. M. \& Beck, A. T. (2004) Cognitive-behavioral therapy for schizophrenia: a review. Journal of Psychiatric Practice 10, 5-16.

Valmaggia, L. R., van der Gaag, M., Tarrier, N., Pijnenborg, M. \& Slooff, C. J. (2005). Cognitive-behavioural therapy for refractory psychotic symptoms of schizophrenia resistant to atypical antipsychotic medication. Randomised controlled trial. British Journal of Psychiatry 186, 324-330.

Volavka, J., Czobor, P., Sheitman, B., Lindenmayer, J. P., Citrome, L., McEvoy, J. P., Cooper, T. B., Chakos, M. \& Lieberman, J. A. (2002). Clozapine, olanzapine, risperidone, and haloperidol in the treatment of patients with chronic schizophrenia and schizoaffective disorder. American Journal of Psychiatry 159, 255-262. 